\title{
Establishment of a model for predicting sentinel lymph node metastasis in early breast cancer based on contrast-enhanced ultrasound and clinicopathological features
}

\author{
Lina Wang ${ }^{1}$, Juntao Li $^{1}$, Jianghua Qiao ${ }^{1}$, Xiaoxia Guo ${ }^{2}$, Xiaolin Bian ${ }^{2}$, Lanwei Guo ${ }^{3}$, Zhenzhen Liu ${ }^{1}$, \\ Zhenduo $\mathrm{Lu}^{1 \wedge}$
}

${ }^{1}$ Department of Breast Surgery, Affiliated Cancer Hospital of Zhengzhou University (Henan Cancer Hospital), Zhengzhou, China; ${ }^{2}$ Department of Ultrasound, Affiliated Cancer Hospital of Zhengzhou University (Henan Cancer Hospital), Zhengzhou, China; ${ }^{3}$ Henan Office for Cancer Control and Research, Affiliated Cancer Hospital of Zhengzhou University (Henan Cancer Hospital), Zhengzhou, China

Contributions: (I) Conception and design: L Wang, Z Liu, Z Lu; (II) Administrative support: None; (III) Provision of study materials or patients: L Wang, Z Lu, X Guo, X Bian; (IV) Collection and assembly of data: L Wang, J Li, J Qiao; L Guo; (V) Data analysis and interpretation: All authors; (VI) Manuscript writing: All authors; (VII) Final approval of manuscript: All authors.

Correspondence to: Zhenzhen Liu. Department of Breast Surgery, Affiliated Cancer Hospital of Zhengzhou University (Henan Cancer Hospital), 127 Dongming Road, Zhengzhou 450008, China. Email: Liuzhenzhen@126.com; Zhenduo Lu. Department of Breast Surgery, Affiliated Cancer Hospital of Zhengzhou University (Henan Cancer Hospital), 127 Dongming Road, Zhengzhou 450008, China. Email: zlyyluzhenduo1145@zzu.edu.cn.

\begin{abstract}
Backgrounda Sentinel lymph node (SLN) biopsy (SLNB) is the standard procedure for axillary staging in clinically node-negative ( $\mathrm{cN} 0$ ) breast cancer patients. However, the positive rate of SLNs among cN0 stage patients is $26-35 \%$. The identification of appropriate candidates for SLNB is quite challenging. This study aimed to establish and verify a predictive model of SLN metastasis using contrast-enhanced ultrasound (CEUS) and other clinicopathological indicators.

Methods: The clinicopathological data of 224 patients who had undergone SLNB at the Affiliated Cancer Hospital of Zhengzhou University from June 2018 to July 2019 were analyzed retrospectively. The risk prediction model of SLN metastasis was established by logistic regression analysis. According to the $\beta$ value of each variable in the model, a risk score system of SLN metastasis was established and verified using the internal population. The predictive model was prospectively applied to 73 patients from July 2019 to September 2019 to evaluate the clinical value of the model in patients with early breast cancer.

Results: Multivariate analysis confirmed that body mass index (BMI), SLN aspect ratio of CEUS mode, SLN aspect ratio of mammography, lympho-vascular invasion, and cytokeratin (CK)5/6 were independent risk factors for SLN metastasis. A scoring system was established according to the above risk factors, and a receiver operating characteristic (ROC) curve was drawn. After internal- and external verification, a corrected ROC curve was drawn, respectively. The ROC curve of the modeling group, internal verification group, and external verification group was 0.9075 (95\% CI: 0.8616-0.9534), 0.8766 (95\% CI: 0.8192-0.9341), and 0.8505 (95\% CI: 0.7333-0.9676), respectively.
\end{abstract}

Conclusions: We constructed and verified a prediction model of SLN metastasis in early breast cancer. The model has a specific predictive value for preoperative evaluation of SLN status.

Keywords: Breast cancer; sentinel lymph node; prediction model; scoring system; contrast-enhanced ultrasound (CEUS)

Submitted Mar 16, 2021. Accepted for publication May 20, 2021.

doi: $10.21037 / g s-21-245$

View this article at: http://dx.doi.org/10.21037/gs-21-245

$\wedge$ ORCID: 0000-0001-6532-9761. 


\section{Introduction}

Sentinel lymph node biopsy (SLNB) has become one of the standard surgical methods for axillary surgery in patients with early invasive breast cancer with negative axillary lymph nodes; it can accurately evaluate the pathological status of axillary lymph nodes $(1,2)$. For patients with negative axillary lymph nodes, SLNB can safely and effectively replace axillary lymph node dissection (ALND) and improve their subsequent quality of life. However, assessment of the indication of SLNB, that is, clinically node-negative (cN0), is still controversial. Previous studies have shown that the positive rate of SLN in patients with clinically negative axillary lymph nodes is about 26-35\% (3-6). Approximately $50 \%$ of patients with positive SLN biopsy findings harbor additional non-sentinel ALN metastases. This possibility represents an important drawback of the SLN biopsy procedure because these patients normally require a second operation (7). More accurate preoperative staging would reduce this need. In clinical practice, examination of the axilla is unsatisfactory for predicting ALN status; we hypothesized that if the model could be used to accurately predict the positive status of axillary SLN before the operation, the positive rate of SLNB might be reduced. It seems impossible that a single examination could identify the status of SLN. In our study, we anticipated the establishment of a clinically acceptable predictive model. The combination of clinical assessment and pathological findings may help to identify patients with positive SLNs and thus avoid SLNB.

In recent years, contrast-enhanced ultrasound (CEUS) using microbubble-based agents has been used for investigation and evaluation of the lymphatic system in both animal and human studies (8). As a new technique of ultrasound (US) imaging, CEUS can obtain more perfusion information, which is helpful for the differential diagnosis of benign and malignant lymph nodes (9). Some previous studies have indicated the value of CEUS enhancing patterns in detecting metastasizing SLNs (10). The diagnostic value can be improved based on conventional US, and the sensitivity, specificity, and accuracy of CEUS in the diagnosis of metastatic SLN can be increased to $84 \%$, $79 \%$, and $80 \%$, respectively (11). Before the introduction of CEUS to routine practice, a study reported that $21 \%$ of patients with a routine axillary US were found to have positive lymph nodes using SLNB, which then necessitated the completion of ALND (12). With improved accuracy of identifying the SLN preoperatively and thus enabling a targeted SLNB, as offered by CEUS, an increasing number of patients were triaged directly to ALND. This resulted in a drop in the number of patients with positive SLNB requiring ALND to $8 \%$ (7). Therefore, we estimate that the inclusion of CEUS in the prediction model will significantly improve its predictive value.

In this study, 224 patients with early breast cancer were recruited from June 2018 to June 2019 in the Affiliated Cancer Hospital of Zhengzhou University. The aim was to analyze and screen the risk factors of axillary SLN metastasis and establish a predictive model of SLN metastasis. Firstly, careful internal verification was carried out. Then, the predictive model was prospectively applied to 73 patients from July 2019 to September 2019 for external verification. Additionally, CEUS of SLN was included in the predictive model for the first time to screen out patients more suitable for SLNB, and reduce the positive rate of clinical SLN. The results of the study were reported as follows. We present the following article in accordance with the STARD reporting checklist (available at http://dx.doi.org/10.21037/ gs-21-245).

\section{Methods}

\section{Patients}

The participants were informed about the purpose of the study, and the study conducted in accordance with the Declaration of Helsinki (as revised in 2013). The study was approved by the Ethics Committee of the Cancer Hospital affiliated to Zhengzhou University (No. 2017407) and informed consent was taken from all the patients. Refer to the indications of sentinel lymph node biopsy in the Chinese Anti-Cancer Association breast cancer diagnosis and treatment guidelines and norms. The entry criteria were as follows: (I) preoperative hollow needle biopsy or local resection biopsy was confirmed as invasive carcinoma of the breast by histopathology. Immunohistochemical (IHC) staining was used to understand the vascular status and to judge whether there was lympho-vascular invasion or not; (II) clinical examination showed that the axillary lymph nodes were negative and no suspicious metastatic lymph nodes were found by routine color Doppler US before the operation; (III) informed signed agreement to use CEUS to evaluate the status of SLN before the operation. The exclusion criteria were as follows: (I) patients with inflammatory breast cancer, as diagnosed by pathology; (II) patients with positive axillary lymph nodes confirmed 
by clinical axillary lymph nodes, or positive axillary lymph nodes confirmed by puncture pathology; (III) pregnant women; (IV) patients who had undergone previous breast surgery or axillary surgery; (V) patients who had received neoadjuvant therapy. Finally, 297 patients were enrolled in the group, including 224 for the training set and 73 for the verification set. The age of the patients was 25-79 years old, and the median age was 50. All 297 cases were unilateral breast lesions, and all of them were treated by operation in our hospital (Figure S2).

\section{CEUS and Surgical management of SLNs}

In this study, the contrast agent used for injection was sulfur hexafluoride microbubble (SonoVue, Bracco Co., MI, Italy). The use of US instruments, setting of contrast conditions, analysis of US images, and interpretation of results were all performed by 2 experienced sonographers (Guo Xiaoxia and Bian Xiaolin), who were blinded to the participants' clinical data. After performing anesthesia in the areola area, $0.5 \mathrm{~mL}$ of contrast medium was injected into the skin around the areola at 3, 6, 9, and 12 o'clock, and the injection area was massaged for 15 seconds. A US probe was used to track the enhanced lymphatic channel from the areola to the axilla. The first enhanced lymph node was recorded as the SLN. Once identified, we labeled the lymphatic channel and SLN on the surface of the skin and recorded the size and number of SLN (Figure S1). If the lymphatic channel or lymph node was not successfully detected, the above process could be repeated. According to the patterns of development, the enhancement patterns of SLN were divided into the following 3 types: (I) homogeneous enhancement: significant and uniform enhancement of the whole lymph node; (II) inhomogeneous enhancement, focal low or no perfusion area in the parenchyma of significant enhancement; (III) weak enhancement or no enhancement: the whole lymph node was weak or showed no enhancement, and the perfusion was homogeneous or inhomogeneous.

Type I was considered as an uninvaded lymph node, type II and type III were considered as suspected metastatic lymph nodes. When the enhancement pattern discovered was inconsistent between the 2 sonographers, a third experienced sonographer made the judgement. Refer to the method of sentinel lymph node biopsy in the Chinese Anti-Cancer Association breast cancer diagnosis and treatment guidelines and norms. The SLNs were located with dye tracer methylene blue. During the operation, any of the following were identified as SLNs: (I) blue-stained lymph nodes; (II) lymph nodes immediately directed by blue-stained lymphatic vessels; (III) suspect lymph nodes that were palpated during operation. The intraoperative resection of SLN was sent for rapid pathological examination. If the pathological results suggested that the lymph node was not invaded, ALND did not go ahead. If the pathological results suggested that the lymph node was invaded, the ALND was proceeded with. If the rapid pathological results suggested that the lymph node was not invaded, but the conventional pathological results suggested that the lymph node was invaded and we need to decide whether to supplement ALND after full communication with the patient.

\section{Statistical analysis}

The data management and all analyses were conducted using SAS statistical software, version 9.4 (SAS Institute Inc., Cary, NC, USA). Univariate analysis was performed by the chi-squared $\left(\chi^{2}\right)$ test. In the univariate analysis, the risk factors of screening variables $(\mathrm{P}<0.05)$ were included in the subsequent multivariate analysis, and the independent risk factors for predicting SLN metastasis were selected $(\mathrm{P}<0.05)$. Following the value of $\beta$ in each variable, the integer was rounded and converted to the corresponding score. The total score was equal to the sum of the scores of each variable. According to the risk score of SLN metastasis in each patient, a receiver operating characteristic (ROC) curve was drawn, and the area under the curve (AUC) and 95\% confidence interval (CI) were calculated. Discrimination of the model, risk scores, and risk categories were assessed by ROC analysis with the leave-one-out cross-validation (LOOCV) method. The Hosmer-Lemeshow goodness of fit statistic was used to assess the reliability of the models, where $\mathrm{P}>0.05$ indicated adequate calibration. According to the ROC curve, the risk of SLN transfer was divided into an average risk group and a high-risk group. According to the risk score of SLN transfer from small to large and stratification, the boundary value of the average risk group and the high-risk group of SLN transfer was determined. Then, the sensitivity and specificity, positive predictive value, and negative predictive value of the SLN metastasis risk scoring system were calculated and verified in the verification group. 


\section{Results}

\section{Clinicopathological features}

A total of 297 female patients were included in the analysis, including 224 cases in the training set and 73 cases in the verification set. The age of participants was $25-79$ years old, and the median age was 50 . All participants underwent SLNB without neoadjuvant therapy before the operation, and the clinicopathological data were complete. Of the 224 patients, 60 were SLN positive, accounting for $26.8 \%$ $(60 / 224)$ of the total, and the proportion of non-SLN negative patients was $56.7 \%(34 / 60)$. Of the 73 participants in the verification set, 14 were SLN positive, accounting for $19.2 \%(14 / 73)$ of the total, and the proportion of nonSLN negative patients was $85.7 \%$ (12/14). In each case, 111 SLN were obtained; 1,016 SLN were collected in the whole group, with an average of 3.42 per case. For the clinical characteristics, the height and weight were calculated according to the formula of body mass index (BMI), and the participants were divided into normal group, overweight group, and obese group according to the adult's obesity standard of the WHO (BMI $\geq 25 \mathrm{~kg} / \mathrm{m}^{2}$ was overweight, $\mathrm{BMI} \geq 30 \mathrm{~kg} / \mathrm{m}^{2}$ was obese). If 1 or more of the first-degree relatives of the participant had cancer, they were defined as having a family history. An estrogen receptor (ER) presence of $>1 \%$ was defined as positive, and a progesterone receptor (PR) presence of $>1 \%$ was defined as positive.

\section{Univariate analysis of axillary sentinel lymph node metastasis}

Univariate analysis showed that the combination of BMI, tumor resection biopsy, tumor size, lympho-vascular invasion, ER status, PR status, CK5/6, epidermal growth factor receptor (EGFR), US lymphatic structure, SLN aspect ratio of US, cortical medulla boundary of lymph nodes, CEUS enhanced mode, SLN aspect ratio of CEUS, and SLN aspect ratio of mammography were potential predictors of SLN metastasis $(\mathrm{P}<0.05$, Table 1, Table S1).

\section{Analysis of multiple factors affecting axillary SLN metastasis}

The results of multivariate logistic regression analysis showed that BMI, SLN aspect ratio of US, CEUS enhanced mode, SLN aspect ratio of mammography, lympho-vascular invasion, and cytokeratin (CK) 5/6 were independent risk factors for SLN metastasis $(\mathrm{P}<0.05$, Table 2).

\section{Establishing a scoring system}

According to the value of the regression coefficient $\beta$ of each variable, the whole number was rounded and converted into a corresponding score. The scoring system based on the $\beta$ value of each variable is shown in Table 3 . The scores ranged from $0-11$, and the total score of each participant was calculated according to the above scoring system. The general situation of the risk score of the study population is shown in Table 4. Among the 224 participants who underwent SLNB, 60 (26.8\%) had SLN metastasis. As shown in Table 4, the transfer rate of SLN with a score of 5 was $23.1 \%$, and that of SLN with a score of 6 was $45.8 \%$. The total detection rate was between 5 and 6 . Therefore, in this study, $0-5$ was defined as average risk and $6-11$ as high risk. This was consistent with the cutoff value calculated by SAS (Figure 1).

\section{Effectiveness evaluation of scoring system model}

The ROC curve of the risk score system of SLN metastasis was drawn according to the risk score of SLN metastasis in each participant. The area under the curve was 0.9075 (95\% CI: 0.8616-0.9534), indicating that the scoring system was good. The result of the Hosmer-Lemeshow goodness-of-fit test showed that the goodness-of-fit of the logistic regression model was good $(\mathrm{P}=0.7375)$. When the cutoff value of 5 points was used for different risks (that is, $\geq 6$ points were judged as patients with SLN metastasis), the test results were further analyzed according to the judgment method of the scoring system. The corresponding sensitivity, specificity, positive predictive value, and negative predictive value were calculated to be $80 \%, 88.4 \%, 71.6 \%$, and $92.4 \%$, respectively.

\section{Verification of the effectiveness of the scoring system}

The remaining cross-validation method was applied to verify the model internally. Among them, N-1 samples (of the $\mathrm{N}$ samples in the data) were trained to predict the probability of SLN transfer of the remaining $\mathrm{N}$ samples, and the corrected ROC curve was drawn. The AUC was 0.8766 (95\% CI: $0.8192-0.9341$ ), and the differentiation of the corrected scoring system was still good (Figure 1). According to the scoring system established by the training set, the corresponding score of each participant 
Table 1 Univariate analysis of clinicopathological indexes and SLN metastasis in patients with breast cancer

\begin{tabular}{|c|c|c|c|c|c|}
\hline Characteristics & Number of cases & \multicolumn{2}{|c|}{ SLN, n (\%) } & $\chi^{2}$ & $P$ value \\
\hline Age, years & & & & 0.341 & 0.871 \\
\hline$\leq 35$ & 18 & $14(77.8)$ & $4(22.2)$ & & \\
\hline $35-50$ & 97 & $72(74.2)$ & $25(25.8)$ & & \\
\hline $\mathrm{BMI}, \mathrm{kg} / \mathrm{m}^{2}$ & & & & 5.267 & 0.022 \\
\hline$<25$ & 136 & $107(78.7)$ & $29(21.3)$ & & \\
\hline$\geq 25$ & 88 & $57(64.8)$ & $31(35.2)$ & & \\
\hline Menopausal status & & & & 0.219 & 0.64 \\
\hline Family history & & & & 0.193 & 0.661 \\
\hline No & 177 & 129 (72.9) & $48(27.1)$ & & \\
\hline Yes & 46 & $35(76.1)$ & $11(23.9)$ & & \\
\hline Tumor location & & & & 1.941 & 0.747 \\
\hline Central & 8 & $7(87.5)$ & $1(12.5)$ & & \\
\hline Outer upper & 127 & $94(74.0)$ & $33(26.0)$ & & \\
\hline Outer lower & 22 & $17(77.3)$ & $5(22.7)$ & & \\
\hline Inner lower & 14 & $9(64.3)$ & $5(35.7)$ & & \\
\hline T1mi & 9 & $9(100.0)$ & $0(0.0)$ & & \\
\hline $\mathrm{T} 1$ & 114 & $87(76.3)$ & $27(23.7)$ & & \\
\hline $\mathrm{T} 2$ & 100 & $68(68.0)$ & $32(32.0)$ & & \\
\hline T3 & 1 & $0(0.0)$ & $1(100.0)$ & & \\
\hline Histologic grade & & & & 4.349 & 0.107 \\
\hline G1 & 11 & $10(90.9)$ & $1(9.1)$ & & \\
\hline G2 & 160 & $108(67.5)$ & $52(32.5)$ & & \\
\hline G3 & 36 & $29(80.6)$ & 7 (19.4) & & \\
\hline Lympho-vascular invasion & & & & 77.254 & $<0.001$ \\
\hline No & 156 & $141(90.4)$ & $15(9.6)$ & & \\
\hline Yes & 68 & $23(33.8)$ & $45(66.2)$ & & \\
\hline
\end{tabular}

Table 1 (continued) 
Table 1 (continued)

\begin{tabular}{|c|c|c|c|c|c|}
\hline Characteristics & Number of cases & \multicolumn{2}{|c|}{ SLN, n (\%) } & $\chi^{2}$ & $P$ value \\
\hline Pathology & & & & 5.121 & 0.148 \\
\hline IDC & 170 & $118(69.4)$ & $52(30.6)$ & & \\
\hline ILC & 10 & $8(80.0)$ & $2(20.0)$ & & \\
\hline Mucinous & 4 & $4(100.0)$ & $0(0.0)$ & & \\
\hline ER Status & & & & 5.569 & 0.018 \\
\hline Positive & 169 & $117(69.2)$ & $52(30.8)$ & & \\
\hline Negative & 55 & $47(85.5)$ & $8(14.5)$ & & \\
\hline Negative & 67 & $56(83.6)$ & $11(16.4)$ & & \\
\hline HER-2 Status & & & & 0.121 & 0.728 \\
\hline Negative & 168 & $124(73.8)$ & $44(26.2)$ & & \\
\hline Positive & 56 & $40(71.4)$ & $16(28.6)$ & & \\
\hline Ki-67 & & & & 0.459 & 0.498 \\
\hline$\leq 14 \%$ & 32 & $25(78.1)$ & 7 (21.9) & & \\
\hline$>14 \%$ & 192 & $139(72.4)$ & $53(27.6)$ & & \\
\hline CK5/6 & & & & 5.126 & 0.036 \\
\hline Number of LN by US & & & & 0.996 & 0.318 \\
\hline 1 & 164 & $123(75.0)$ & $41(25.0)$ & & \\
\hline 2 & 60 & $41(68.3)$ & $19(31.7)$ & & \\
\hline US Iymphatic structure & & & & 24.282 & $<0.001$ \\
\hline Yes & 192 & $152(79.2)$ & $40(20.8)$ & & \\
\hline No & 32 & $12(37.5)$ & $20(62.5)$ & & \\
\hline SLN aspect ratio of US & & & & 12.406 & $<0.001$ \\
\hline$\leq 2$ & 117 & $74(63.2)$ & $43(36.8)$ & & \\
\hline$>2$ & 107 & $90(84.1)$ & $17(15.9)$ & & \\
\hline
\end{tabular}

Table 1 (continued) 
Table 1 (continued)

\begin{tabular}{|c|c|c|c|c|c|}
\hline Characteristics & Number of cases & \multicolumn{2}{|c|}{ SLN, n (\%) } & $\chi^{2}$ & $P$ value \\
\hline Cortical medulla boundary & & & & 18.137 & $<0.001$ \\
\hline Clear & 179 & $143(79.4)$ & $37(20.6)$ & & \\
\hline Lack of clarity & 44 & $21(47.7)$ & $23(52.3)$ & & \\
\hline 0 & 2 & $0(0.0)$ & $2(100)$ & & \\
\hline 1 & 127 & $96(75.6)$ & $31(24.4)$ & & \\
\hline 2 & 74 & $53(71.6)$ & $21(28.4)$ & & \\
\hline 3 & 21 & $15(71.4)$ & $6(28.6)$ & & \\
\hline II/III & 70 & $31(44.3)$ & $39(55.7)$ & & \\
\hline SLN aspect ratio of CEUS & & & & 3.275 & 0.07 \\
\hline$\leq 2$ & 131 & $90(68.7)$ & $41(31.3)$ & & \\
\hline$>2$ & 93 & $74(79.6)$ & $19(20.4)$ & & \\
\hline SLN aspect ratio of mammography & & & & 8.856 & 0.001 \\
\hline$\leq 2$ & 212 & $160(75.5)$ & $52(24.5)$ & & \\
\hline$>2$ & 12 & 4 (33.3) & $8(66.7)$ & & \\
\hline
\end{tabular}

*: $\mathrm{T} \leq 0.1 \mathrm{~cm}$ is $\mathrm{T} 1 \mathrm{mi}, 0.1 \mathrm{~cm}<\mathrm{T} \leq 2 \mathrm{~cm}$ is $\mathrm{T} 1,2 \mathrm{~cm}<\mathrm{T} \leq 5 \mathrm{~cm}$ is $\mathrm{T} 2, \mathrm{~T}>5 \mathrm{~cm}$ is $\mathrm{T} 3$; \#: histological grade was missing in 17 participants. BMI, body mass index; IDC, invasive ductal carcinoma; ILC, invasive lobular carcinoma; DCIS-IDC, ductal carcinoma in situ- invasive ductal carcinoma; ER/PR, estrogen/progesterone receptor; HER-2, human epidermal growth factor receptor-2; EGFR, epidermal growth factor receptor; US, ultrasound; SLN, sentinel lymph node; CEUS, contrast-enhanced ultrasonography.

Table 2 Logistics regression multivariate analysis of SLN metastasis of breast cancer

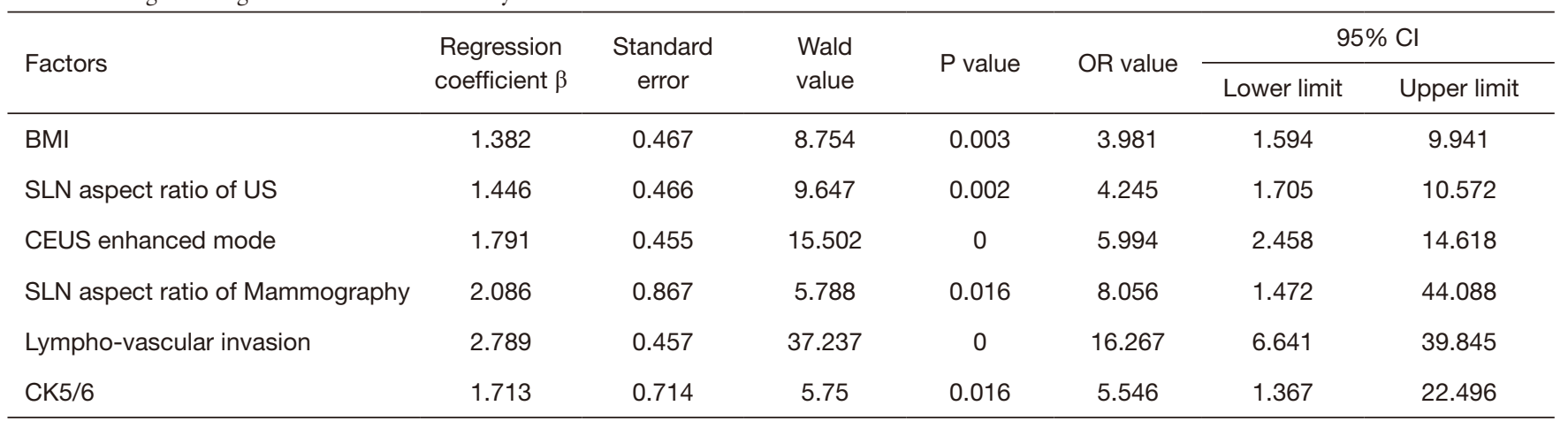

SLN, sentinel lymph node; OR, odds ratio; Cl, confidence interval; BMI, body mass index; CEUS, contrast-enhanced ultrasound; CK5/6, cytokeratin 5/6. 
Table 3 Scoring system

\begin{tabular}{|c|c|}
\hline Exposure factors & Score \\
\hline \multicolumn{2}{|l|}{ BMI } \\
\hline$<25$ & 0 \\
\hline$\geq 25$ & 1 \\
\hline \multicolumn{2}{|c|}{ SLN aspect ratio of US } \\
\hline$\leq 2$ & 1 \\
\hline$>2$ & 0 \\
\hline \multicolumn{2}{|c|}{ CEUS enhanced mode } \\
\hline I & 0 \\
\hline II/III & 2 \\
\hline \multicolumn{2}{|c|}{ SLN aspect ratio of Mammography } \\
\hline$\leq 2$ & 2 \\
\hline$>2$ & 0 \\
\hline \multicolumn{2}{|c|}{ Lympho-vascular invasion } \\
\hline No & 0 \\
\hline Yes & 3 \\
\hline \multicolumn{2}{|l|}{$\mathrm{CK} 5 / 6$} \\
\hline Negative & 2 \\
\hline Positive & 0 \\
\hline
\end{tabular}

BMI, body mass index; SLN, sentinel lymph node; CEUS, contrast-enhanced ultrasound; CK5/6, cytokeratin 5/6.

in the verification set was calculated, and the ROC curve of the SLN metastasis risk scoring system was drawn. The AUC was 0.8505 (95\% CI: 0.7333-0.9676), and the differentiation of the scoring system of the verification set was still good (Figure 2). According to the above risk grouping criteria, there were 57 cases in the average-risk group, with 3 cases $(5.3 \%)$ in the SLN transfer group, and 16 cases in the high-risk group, with 11 cases $(68.8 \%)$ in the SLN transfer group. The sensitivity, specificity, positive predictive value, and negative predictive value of the scoring system were $68.8 \%, 94.7 \%, 78.6 \%$, and $91.5 \%$, respectively.

\section{Discussion}

The use of SLNB has been a milestone in the field of surgical treatment of breast cancer in the 20th century. For patients with negative SLN, avoiding ALND has been recommended by various guidelines, and has become the
Table 4 General situation of risk score of early breast cancer population

\begin{tabular}{lccc}
\hline \multirow{2}{*}{ Score } & \multirow{2}{*}{$\begin{array}{c}\text { Number of } \\
\text { cases }\end{array}$} & $(-)$ & $(+)$ \\
\cline { 3 - 4 } & 5 & $5(100 \%)$ & $0(0 \%)$ \\
1 & 9 & $9(100 \%)$ & $0(0 \%)$ \\
2 & 36 & $35(97.2 \%)$ & $1(2.8 \%)$ \\
3 & 64 & $60(93.8 \%)$ & $4(6.3 \%)$ \\
4 & 30 & $26(86.7 \%)$ & $4(13.3 \%)$ \\
5 & 13 & $10(76.9 \%)$ & $3(23.1 \%)$ \\
6 & 24 & $13(54.2 \%)$ & $11(45.8 \%)$ \\
7 & 11 & $4(36.4 \%)$ & $7(63.6 \%)$ \\
8 & 19 & $1(5.3 \%)$ & $18(94.7 \%)$ \\
9 & 11 & $1(9.1 \%)$ & $10(90.9 \%)$ \\
10 & 1 & $0(0 \%)$ & $1(100 \%)$ \\
11 & 1 & $0(0 \%)$ & $1(100 \%)$ \\
\hline
\end{tabular}

SLN, sentinel lymph node.

current standard mode of treatment. With the continuous development of medical social demographics, the proportion of patients with early breast cancer is constantly increasing, and axillary lymph node-negative breast cancer accounts for more than $50 \%$ of new cases. For patients with negative palpation of axillary lymph nodes, the positive rate of SLN was $26-35 \%$. Among patients with negative axillary lymph nodes in the National Surgical Adjuvant Breast and Bowel Project (NSABP)-32 multicenter test, the positive rate of SLN was $26 \%$ (13). The data of our hospital were similar, between $27 \%$ and $31 \%(3,14)$. Therefore, how to accurately judge the indication of SLNB and reduce the positive rate of SLN was a great challenge to our research. Our belief is that SLNB is unlikely to be replaced by other noninvasive or less invasive methods at present due to the gold standard role of histopathology. Consequently, we aimed to assess whether the established model could be utilized to noninvasively predict SLN metastasis and identify SLNB candidates, rather than the feasibility of SLNB replaced with predictive models. This study attempted to establish an effective prediction model of axillary SLN metastasis. Direct ALND can avoid the wastage of medical resources on patients with a high probability of SLN metastasis. In contrast to the previous prediction models of SLN status, we were the first to include SLN contrast echocardiography 


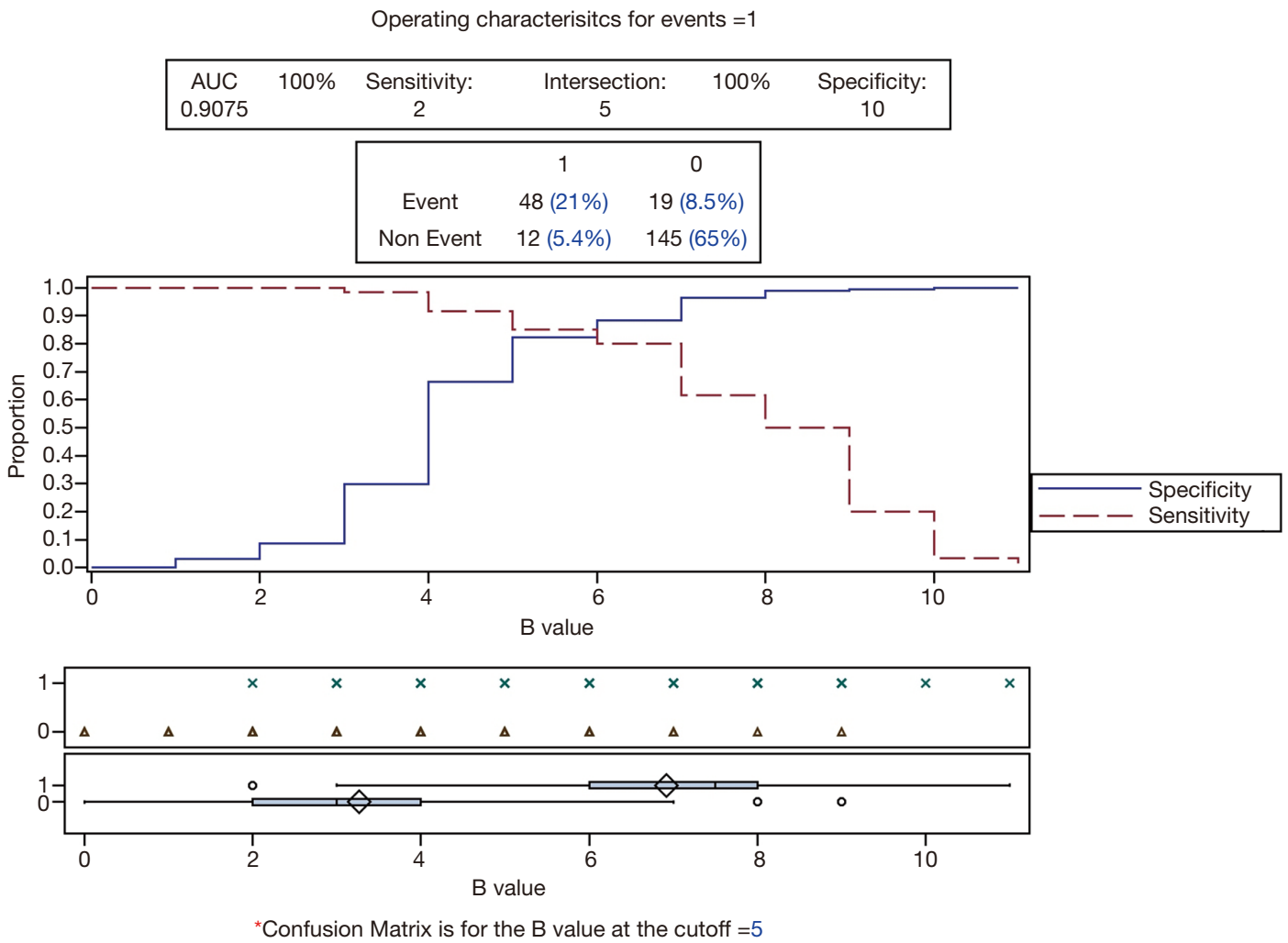

Figure 1 The cutoff value calculated by SAS based on the $\beta$ value. Cutoff value of sentinel lymph node metastasis risk is 5 . 0-5 is defined as average risk and 6-11 as high risk.

A

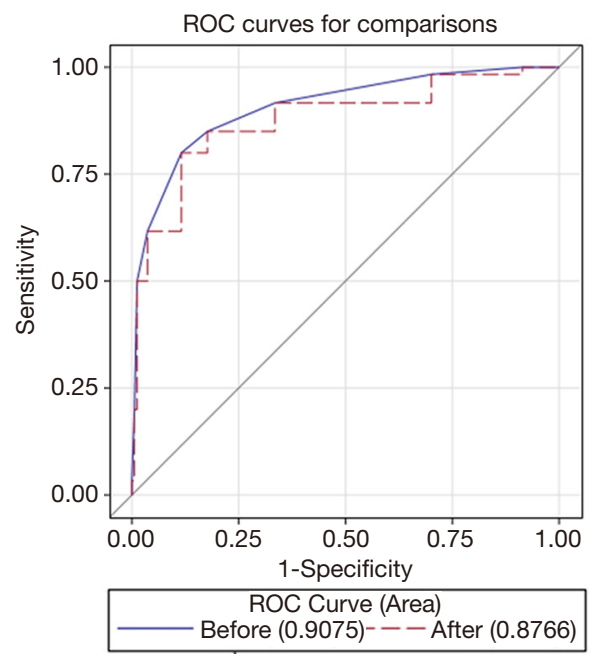

B

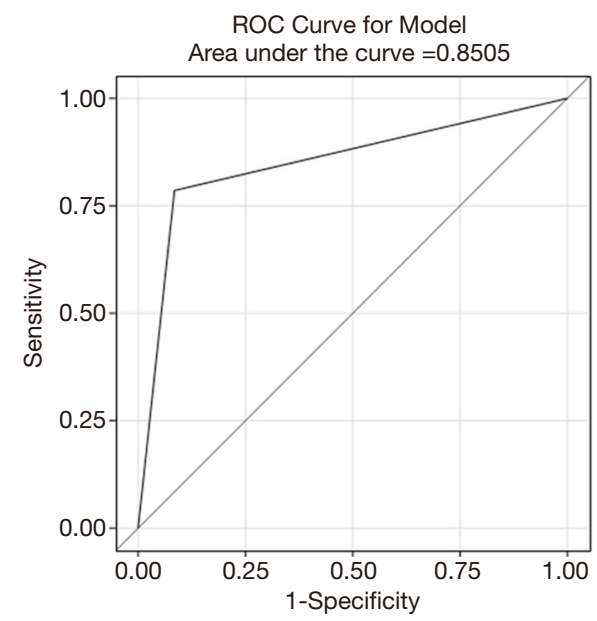

Figure 2 The ROC curve of the modeling group, internal verification group, and external verification group. (A) ROC curve before and after correction; (B) ROC curve of the verification group. ROC, receiver operating characteristic. 
as a predictive factor in the model.

Metastasis of the SLN is closely related to many factors, and clinicopathological features may be involved in the occurrence and development of SLN metastasis. Most studies have shown that age, size of the primary tumor, and histological grade are independent factors affecting SLN metastasis (15). In this study, BMI, SLN aspect ratio, CEUS enhancement model, SLN molybdenum target length-todiameter ratio, vascular tumor thrombus, and CK5/6 were independent risk factors for SLN metastasis.

In this study, the risk of SLN metastasis in overweight and obese patients was higher than that in patients of normal weight. We know that breast cancer is characterized by its occurrence and growth in adipose tissue, which provides structural and paracrine support for tumor growth. Studies have shown that adipose tissue cells can be induced by tumor cells, around which a large number of growth factors, chemokines, and matrix-degrading enzymes are produced, which is beneficial to the proliferation and invasion of tumor cells $(16,17)$. Previous studies have shown that with the rise in the level of excess fat synthesis and secretion of leptin in women, the probability of highly malignant and poorly differentiated breast cancer is greatly increased (18).

The risk of sentinel metastasis was significantly increased in patients with an axillary lymph node aspect ratio $\leq 2$ under US, and the morphological changes of axillary lymph nodes were an important index of metastasis (11). The results of the univariate analysis showed that the aspect ratio of the SLN was related to the metastatic status of the SLN, and the results of multivariate analysis showed that the aspect ratio of the lymph node was an independent risk factor for the SLN. That is, the greater the aspect ratio of lymph nodes, the smaller the probability of lymph node metastasis. Although the presence or absence of the hilar structure of the lymph node and the clarity of the cortical medulla are related to the status of SLN metastasis, they were not identified as independent risk factors. The principle of the aspect ratio of lymph nodes in mammography is consistent with the aspect ratio of lymph nodes under US.

The new technology of CEUS has been developed and perfected continuously in recent years. The principle of CEUS is to sharpen the contrast within the tissues and increase the display of tissues, organs, and lesions by injecting contrast media through different pathways. Examination with CEUS is a new proposal for finding and predicting metastasis of breast cancer SLN. The data of our center showed that the sensitivity, specificity, and accuracy of CEUS for preoperative evaluation of SLN were $80.8 \%, 86.0 \%$, and $84.3 \%$, respectively, suggesting that preoperative CEUS has high clinical value in predicting metastasis of SLN (19). Additionally, CEUS can provide preoperative SLN location information, and using CEUS to trace SLN has a high detection rate and accuracy. In this study, CEUS showed that the risk of SLN metastasis in inhomogeneous enhanced type II/III mode was 5.994 times higher than that in uniformly enhanced type I mode, which was higher than the predictive value of conventional US. Among the participants with negative SLN predicted by conventional US, $21 \%$ of lymph nodes were positive after SLN biopsy, and these participants finally underwent ALND. Improved accuracy of identifying the SLN preoperatively was offered by CEUS. Thus, a targeted SLNB was enabled, and an increasing number of patients were consequentially triaged directly to ALND. This led to a drop in the number of SLNB-positive patients who needed ALND to $8 \%$ (7). Therefore, adding CEUS to the prediction model can further improve the predictive value of the model. However, compared with the vascular infiltration with an odds ratio (OR) of 16.267, its predictive value is on the low side. The reason is that metastatic lymph nodes may show enhanced and diffuse diversity after radiography, and the distribution density of peripheral blood vessels also affects the judgment of sonographers. Therefore, the diagnostic stability of CEUS needs some consensus and time criteria to guide the evaluation, so as to make the subjective judgment more objective (20).

Lympho-vascular invasion is a general term for lymphatic infiltration and vascular infiltration of the tumor. The presence of a lympho-vascular tumor thrombus demonstrates that there is lymphatic and vascular infiltration, even if the SLN is negative (21). The local, regional, and distant recurrence rate of lympho-vascular tumor thrombi were higher than that of non-lymphovascular tumor thrombi. The results of univariate analysis showed that the status of SLN metastasis was related to the lympho-vascular invasion, and the difference between the 2 groups was statistically significant. Participants with lympho-vascular invasion were more likely to have SLN metastasis. Further multivariate analysis also showed that the presence of lympho-vascular invasion was an independent risk factor for SLN metastasis. The risk of SLN metastasis in participants with lympho-vascular invasion was higher than that in those without lymphovascular invasion. The conclusion of this study was similar to that reported in the literature, but the OR value was 
higher than that of other studies.

The marker CK5/6 is an important for the IHC diagnosis of basal breast cancer. The positive expression rate of CK5/6 was $15.6 \%$ in this study. The positive expression rate of CK5 in triple-negative breast cancer (TNBC) was $72.4 \%$. The positive expression of CK5 was the protective factor of SLN metastasis. Previous studies have shown that TNBC patients with CK5/6 negative expression were more likely to develop early micro-metastasis (22). The studies by Adamo et al. and Maeda et al. suggested that the recurrencefree survival and overall survival of TNBC patients with loss of CK5/6 expression were worse than CK5/6-positive $(23,24)$. It can be speculated that CK5/6-negative patients are more likely to have SLN metastasis.

The scoring system based on the above risk factors had a high accuracy in predicting the risk of SLN metastasis in early breast cancer and had a certain predictive value for preoperative evaluation of SLN status. We carried out careful internal and external verification, the results supported the stability of the model, and allowed better confirmation of the practicability of the prediction model as a guidance tool. A major limitation of our study was that the design was a single-center retrospective analysis, which may not be consistent with the multicenter design; another was that of the sample size being small. In the future, multicenter, randomized controlled, and larger samples of prospective studies are needed to further verify our findings.

\section{Conclusions}

Based on the results of multivariate analysis, we established a predictive model that can be used as an auxiliary tool to screen high-risk and low-risk patients with early breast cancer SLN metastasis, and then to determine surgical treatment according to the characteristics of the patient's condition. In future research, it is important for us to work in combination with other diagnosis and treatment centers to re-evaluate the factors related to SLN metastasis, improve the predictive model, optimize the variables in the model, and make preoperative prediction and prognosis evaluation more convenient and feasible.

\section{Acknowledgments}

Funding: This study was supported by the Henan Provincial Medical Science and Technology Research Project (201702250).

\section{Footnote}

Reporting Checklist: The authors have completed the STARD reporting checklist. Available at http://dx.doi.org/10.21037/ gs-21-245

Data Sharing Statement: Available at http://dx.doi. org/10.21037/gs-21-245

Conflicts of Interest: All authors have completed the ICMJE uniform disclosure form (available at http://dx.doi. org/10.21037/gs-21-245). The authors have no conflicts of interest to declare.

Ethical Statement: The authors are accountable for all aspects of the work in ensuring that questions related to the accuracy or integrity of any part of the work are appropriately investigated and resolved. The study was approved by the Ethics Committee of the Cancer Hospital affiliated to Zhengzhou University (No.2017407), was conducted in accordance with the Declaration of Helsinki (as revised in 2013). Informed consent was taken from all the patients.

Open Access Statement: This is an Open Access article distributed in accordance with the Creative Commons Attribution-NonCommercial-NoDerivs 4.0 International License (CC BY-NC-ND 4.0), which permits the noncommercial replication and distribution of the article with the strict proviso that no changes or edits are made and the original work is properly cited (including links to both the formal publication through the relevant DOI and the license). See: https://creativecommons.org/licenses/by-nc-nd/4.0/.

\section{References}

1. Chatterjee A, Serniak N, Czerniecki B. Sentinel lymph node biopsy in breast cancer: a work in progress. Cancer J 2015;21:7-10.

2. Qiu SQ, Zhang GL, Jansen L, et al. Evolution in sentinel lymph node biopsy in breast cancer. Crit Rev Oncol Hematol 2018;123:83-94.

3. Hou YY, Jiao DC, Qiao JH, et al. Analysis of predictive value of ultrasonography combined with clinical pathological factors in sentinel lymph node status of breast cancer. Chinese Journal of Cancer Prevention and Treatment 2017;24:1723-7.

4. Fleissig A, Fallowfield L, Langridge C, et al. Post- 
operative arm morbidity and quality of life. Results of the ALMANAC randomised trial comparing sentinel node biopsy with standard axillary treatment in the management of patients with early breast cancer. Breast Cancer Res Treat 2006;95:279-93.

5. Veronesi U, Paganelli G, Viale G, et al. A randomized comparison of sentinel-node biopsy with routine axillary dissection in breast cancer. New Engl J Med 2003;349:546-53.

6. Veronesi U, Paganelli G, Viale G, et al. Sentinel-lymphnode biopsy as a staging procedure in breast cancer: update of a randomised controlled study. Lancet Oncol 2006;7:983-90.

7. Sever AR, Mills P, Weeks J, et al. Preoperative needle biopsy of sentinel lymph nodes using intradermal microbubbles and contrast-enhanced ultrasound in patients with breast cancer. AJR Am J Roentgenol 2012;199:465-70.

8. Sever AR, Mills P, Jones SE, et al. Sentinel node identification using microbubbles and contrast-enhanced ultrasonography. Clin Radiol 2012;67:687-94.

9. Dellaportas D, Koureas A, Contis J, et al. ContrastEnhanced Color Doppler Ultrasonography for Preoperative Evaluation of Sentinel Lymph Node in Breast Cancer Patients. Breast Care (Basel) 2015;10:331-5.

10. Zhao J, Zhang J, Zhu Q, et al. The value of contrastenhanced ultrasound for sentinel lymph node identification and characterisation in pre-operative breast cancer patients: A prospective study. Eur Radiol 2018;28:1654-61.

11. Matsuzawa F, Einama T, Abe H, et al. Accurate diagnosis of axillary lymph node metastasis using contrastenhanced ultrasonography with Sonazoid. Mol Clin Oncol 2015;3:299-302.

12. Mills P, Sever A, Weeks J, et al. Axillary ultrasound assessment in primary breast cancer: an audit of 653 cases. Breast J 2010;16:460-3.

13. Krag DN, Anderson SJ, Julian TB, et al. Sentinel-lymphnode resection compared with conventional axillarylymph-node dissection in clinically node-negative patients with breast cancer: overall survival findings from the NSABP B-32 randomised phase 3 trial. Lancet Oncol 2010;11:927-33.

14. Li JT, Zhao HM, Guo XH, et al. Preoperative evaluation of sentinel lymph node biopsy using contrast-enhanced ultrasonography in early breast cancer patients and the involved disturbing factors. Zhonghua Yi Xue Za Zhi 2019;99:1086-9.
15. Liu C, Zhao Z, Gu X, et al. Establishment and Verification of a Bagged-Trees-Based Model for Prediction of Sentinel Lymph Node Metastasis for Early Breast Cancer Patients. Front Oncol 2019;9:282.

16. Chu DT, Phuong TNT, Tien NLB, et al. The Effects of Adipocytes on the Regulation of Breast Cancer in the Tumor Microenvironment: An Update. Cells 2019;8:857.

17. Strong AL, Ohlstein JF, Biagas BA, et al. Leptin produced by obese adipose stromal/stem cells enhances proliferation and metastasis of estrogen receptor positive breast cancers. Breast Cancer Res 2015;17:112.

18. Newman G, Gonzalez-Perez R. Leptin-cytokine crosstalk in breast cancer. Mol Cell Endocrinol 2014;382:570-82.

19. Bailey A, Layne G, Shahan C, et al. Comparison between Ultrasound and Pathologic Status of Axillary Lymph Nodes in Clinically Node-negative Breast Cancer Patients. Am Surg 2015;81:865-9.

20. Yuan Z, Quan J, Yunxiao Z, et al. Diagnostic value of contrast-enhanced ultrasound parametric imaging in breast tumors. J Breast Cancer 2013;16:208-13.

21. Hwang KT, Kim YA, Kim J, et al. The influences of peritumoral lymphatic invasion and vascular invasion on the survival and recurrence according to the molecular subtypes of breast cancer. Breast Cancer Res Treat 2017;163:71-82.

22. Ivković-Kapicl T, Panjković M, Nikolić I, et al. Expression of cytokeratins 5/6 and cytokeratin 17 in invasive breast carcinoma. Vojnosanit Pregl 2012;69:1031-8.

23. Adamo B, Ricciardi G, Ieni A, et al. The prognostic significance of combined androgen receptor, E-Cadherin, Ki67 and CK5/6 expression in patients with triple negative breast cancer. Oncotarget 2017;8:76974-86.

24. Maeda T, Nakanishi Y, Hirotani Y, et al. Immunohistochemical co-expression status of cytokeratin $5 / 6$, androgen receptor, and p53 as prognostic factors of adjuvant chemotherapy for triple negative breast cancer. Med Mol Morphol 2016;49:11-21.

(English Language Editor: J. Jones)

Cite this article as: Wang L, Li J, Qiao J, Guo X, Bian X, Guo L, Liu Z, Lu Z. Establishment of a model for predicting sentinel lymph node metastasis in early breast cancer based on contrast-enhanced ultrasound and clinicopathological features. Gland Surg 2021;10(5):1701-1712. doi:10.21037/gs-21-245 

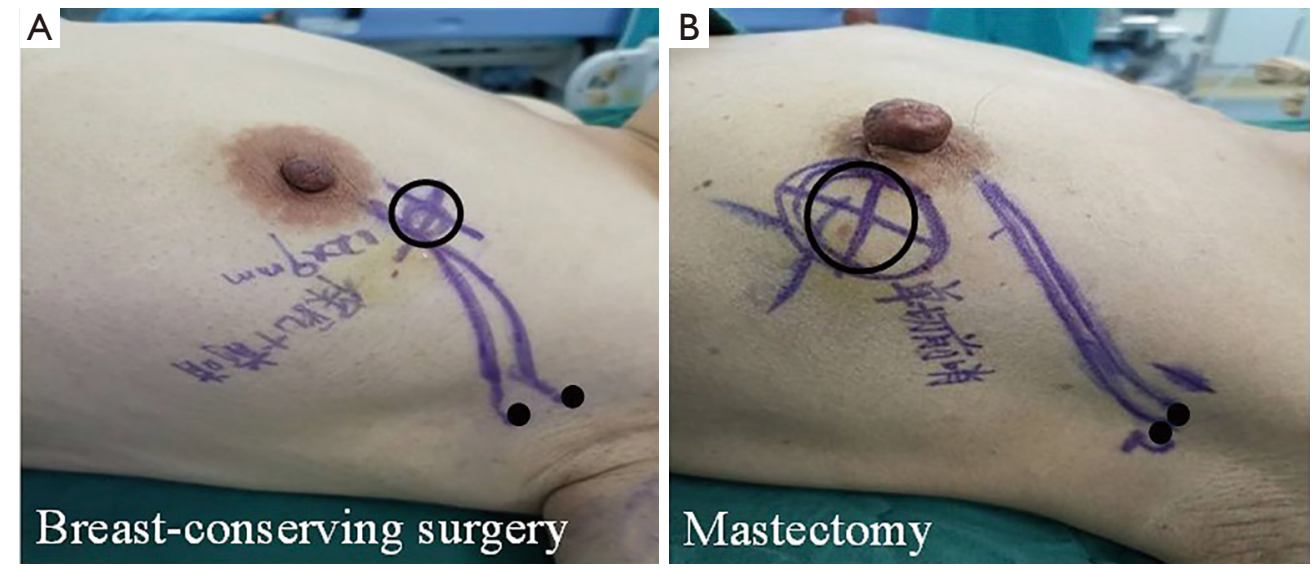

Figure S1 Body surface identification of sentinel lymph node biopsy before operation. (A)॰ represent the location of the tumor, $\bullet$ represent the location of SLN, and represents the pathway of lymphatic drainage; (B)○ represent the location of the tumor, $\bullet$ represent the location of SLN, and $\sim$ represents the pathway of lymphatic drainage.SLN, sentinel lymph node.

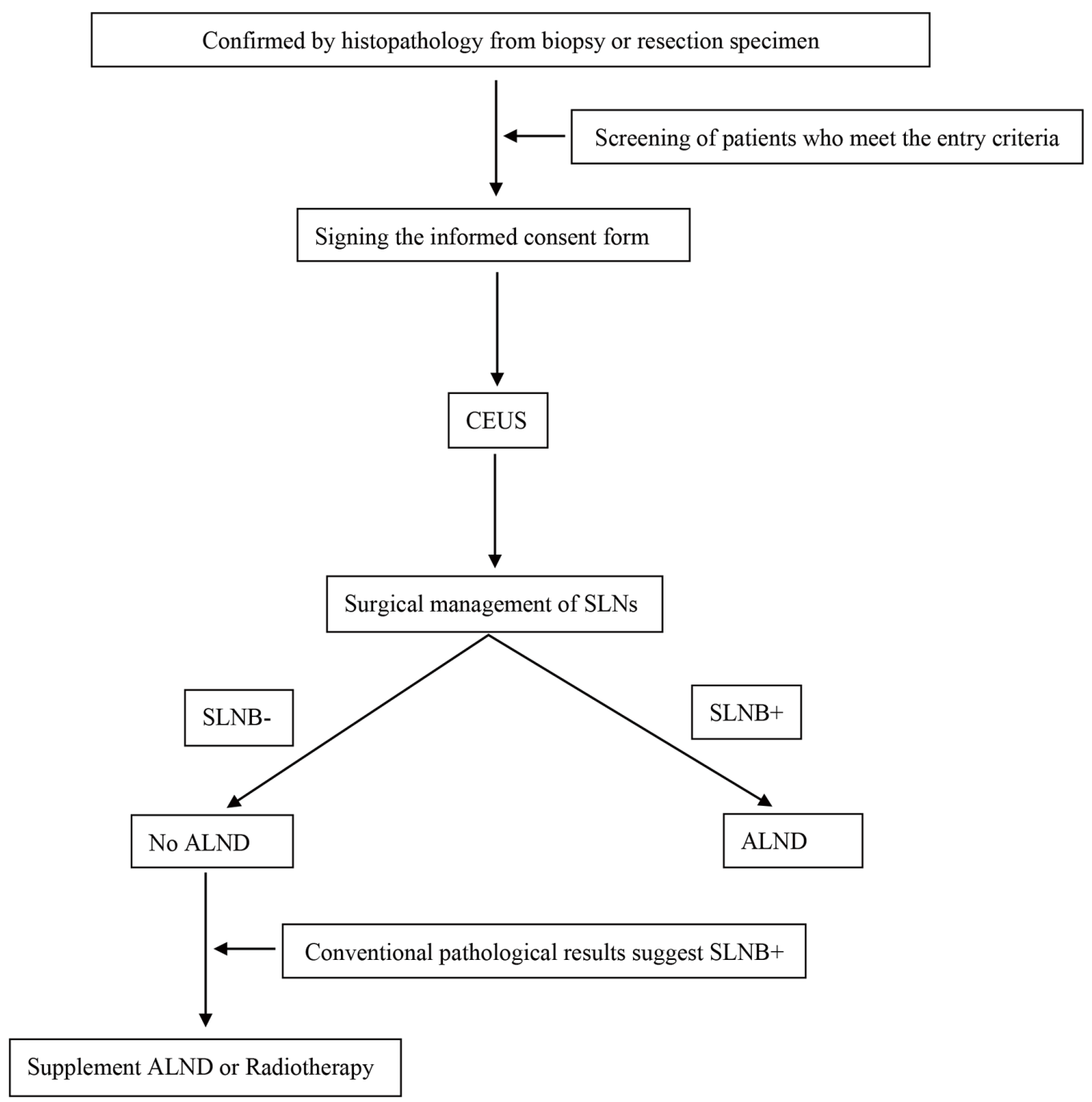

Figure S2 Flow of participants. CEUS, contrast-enhanced ultrasonography; SLNB, sentinel lymph node biopsy; ALND, axillary lymph node dissection. 
Table S1 The clinicopathological Indexes of 73 patients with breast cancer in the external verification group

\begin{tabular}{|c|c|c|c|}
\hline \multirow{2}{*}{ Characteristics } & \multirow{2}{*}{ No. of cases } & \multicolumn{2}{|c|}{ SLN, n (\%) } \\
\hline & & $(-)$ & $(+)$ \\
\hline \multicolumn{4}{|l|}{ Age } \\
\hline$\leq 35$ & 5 & $5(100.0)$ & $0(0)$ \\
\hline $35-50$ & 29 & $22(75.9)$ & $7(24.1)$ \\
\hline$>50$ & 39 & $31(79.5)$ & $8(20.5)$ \\
\hline \multicolumn{4}{|l|}{ BMI } \\
\hline$<25$ & 45 & $37(82.2)$ & $8(17.8)$ \\
\hline$\geq 25$ & 28 & $21(75.0)$ & $7(25.0)$ \\
\hline Menopausal status & & & \\
\hline Pre-menopausal & 45 & $39(86.7)$ & $6(13.3)$ \\
\hline Post-menopausal & 28 & $19(67.9)$ & $9(32.1)$ \\
\hline Family history & & & \\
\hline No & 57 & $45(78.9)$ & $12(21.1)$ \\
\hline Yes & 16 & $13(81.2)$ & $3(18.8)$ \\
\hline Tumor location & & & \\
\hline Central & 4 & $4(100)$ & $0(0)$ \\
\hline Outer upper & 43 & $34(79.1)$ & $9(20.9)$ \\
\hline Outer lower & 11 & $7(63.6)$ & $4(36.4)$ \\
\hline Inner lower & 3 & $3(100)$ & $0(0)$ \\
\hline Inner upper & 12 & $10(83.3)$ & $2(16.7)$ \\
\hline Excision biopsy & & & \\
\hline No & 59 & $46(78.0)$ & $13(22.0)$ \\
\hline Yes & 14 & $12(85.7)$ & $2(14.3)$ \\
\hline Tumor size ${ }^{*}$ & & & \\
\hline T1mi & 5 & $5(100)$ & $0(0)$ \\
\hline $\mathrm{T} 1$ & 31 & 20 (64.5) & $11(35.5)$ \\
\hline $\mathrm{T} 2$ & 37 & $30(81.1)$ & $7(18.9)$ \\
\hline Histologic grade" & & & \\
\hline G1 & 9 & $8(88.9)$ & $1(11.1)$ \\
\hline G2 & 53 & $41(77.4)$ & $12(22.6)$ \\
\hline G3 & 11 & $9(81.8)$ & $2(22.2)$ \\
\hline Lympho-vascular inv & & & \\
\hline No & 55 & $51(94.4)$ & $4(7.3)$ \\
\hline Yes & 18 & $7(38.9)$ & $11(61.1)$ \\
\hline Pathology & & & \\
\hline IDC & 46 & $37(80.4)$ & $9(19.6)$ \\
\hline ILC & 3 & $3(100.0)$ & $0(0)$ \\
\hline DCIS-IDC & 22 & $17(77.3)$ & $5(22.7)$ \\
\hline Mucinous & 2 & $1(50.0)$ & $1(50.0)$ \\
\hline ER Status & & & \\
\hline Positive & 56 & $43(76.8)$ & $13(23.2)$ \\
\hline Negative & 17 & $15(88.2)$ & $2(11.8)$ \\
\hline PR status & & & \\
\hline Positive & 47 & $38(80.9)$ & $9(19.1)$ \\
\hline Negative & 26 & $20(76.9)$ & $6(23.1)$ \\
\hline HER-2 Status & & & \\
\hline Negative & 49 & $40(81.6)$ & $9(18.4)$ \\
\hline Positive & 24 & $18(75.0)$ & $6(25.0)$ \\
\hline Ki-67 & & & \\
\hline$\leq 14 \%$ & 16 & $14(87.5)$ & $2(12.5)$ \\
\hline$>14 \%$ & 57 & $44(77.2)$ & $13(22.8)$ \\
\hline CK5/6 & & & \\
\hline Negative & 19 & $16(84.2)$ & $3(15.8)$ \\
\hline Positive & 54 & $42(77.8)$ & $12(22.2)$ \\
\hline EGFR & & & \\
\hline Negative & 49 & $40(81.6)$ & $9(18.4)$ \\
\hline Positive & 24 & $18(75.0)$ & $6(25.0)$ \\
\hline Number of $L N$ by US & & & \\
\hline 1 & 54 & $42(77.8)$ & $12(22.2)$ \\
\hline 2 & 19 & $16(84.2)$ & $3(15.8)$ \\
\hline US Iymphatic structu & & & \\
\hline Yes & 71 & 57 (80.3) & 14 (19.7) \\
\hline No & 2 & $1(50.0)$ & $1(50.0)$ \\
\hline$S L N$ aspect ratio of $L$ & & & \\
\hline$\leq 2$ & 23 & $17(73.9)$ & $6(26.1)$ \\
\hline$>2$ & 50 & $41(82.0)$ & $9(18.0)$ \\
\hline Cortical medulla bou & & & \\
\hline Clear & 54 & $42(77.8)$ & $12(22.2)$ \\
\hline Lack of clarity & 19 & $16(84.2)$ & $3(15.8)$ \\
\hline Number of $L N$ by $C E$ & & & \\
\hline 0 & 6 & $2(33.3)$ & $4(66.7)$ \\
\hline 1 & 49 & $41(83.7)$ & $8(16.3)$ \\
\hline 2 & 16 & $14(87.5)$ & $2(12.5)$ \\
\hline 3 & 2 & $1(50.0)$ & $1(50.0)$ \\
\hline CEUS enhanced moc & & & \\
\hline 1 & 53 & $47(88.7)$ & $6(11.3)$ \\
\hline$\|/\|$ & 20 & $11(55.0)$ & $9(45.0)$ \\
\hline$S L N$ aspect ratio of $C$ & & & \\
\hline$\leq 2$ & 38 & $27(71.1)$ & $11(28.9)$ \\
\hline$>2$ & 35 & $31(88.6)$ & $4(11.4)$ \\
\hline SLN aspect ratio of $n$ & & & \\
\hline$\leq 2$ & 48 & $44(91.7)$ & $4(8.3)$ \\
\hline$>2$ & 25 & $14(56.0)$ & $11(44.0)$ \\
\hline
\end{tabular}

: $T \leq 0.1 \mathrm{~cm}$ is $T 1 \mathrm{mi}, 0.1 \mathrm{~cm}<\mathrm{T} \leq 2 \mathrm{~cm}$ is $T 1,2 \mathrm{~cm}<\mathrm{T} \leq 5 \mathrm{~cm}$ is $T 2, T>5 \mathrm{~cm}$ is $\mathrm{T}$; \#: histological grade was missing in 17 participants. BMI, body mass index; IDC, invasive ductal carcinoma; ILC, invasive lobular carcinoma; DCIS-IDC, ductal carcinoma in situ- invasive

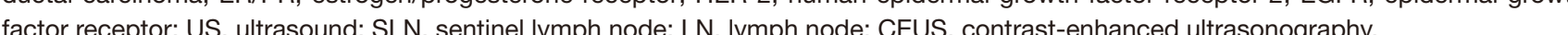

